\title{
New Optical Transforms for Pattern Recognition
}

\author{
DAVID CASASENT SENIOR MEMBER, IEEE AND DEMETRI PSALTIS
}

\begin{abstract}
New optical transformations are discussed. These include Mellin transforms, Fourier-Mellin transforms, and combinations of geometrical transformations and Fourier-Mellin operntions. Scale, positional, and rotational invariant correlations with no loss in SNR are demonstrated.
\end{abstract}

\section{INTRODUCTION}

T HE BASIC operation performed in a coherent optical processor is the Fourier transform. When properly synthesized matched spatial filters are used, the convolution and correlation can be realized. These operations still rely on the Fourier transform property of a lens. To extend the operations possible in an optical computer, considerable attention has recently focused on geometrical transformations [1], image preprocessing by halftone screens [2], nonlinear operations [3], [4], optical feedback [5], hybrid processors [6]-[8], generalized optical operations [9], [10], and other approaches. These have been summarized in this issue [11] and elsewhere.

The two-dimensional correlation is one of the most powerful operations possible in a coherent optical processor. When performed in real time on multiple Vander Lugt spatial filters [12], the computing power of such a system far exceeds that of a digital computer. One reason for the lack of commercial optical pattern-recognition system is the need for a real-time input and filter plane transducer [13]. Another reason is the reliance of optical processors on the Fourier transform operation and conventional matched spatial filters. Specifically, if the input differs in scale or orientation from the object used to form the matched filter, a severe loss in the signal-to-noise ratio (SNR) of the correlation peak occurs. Experimentally obtained SNR variations for a moderate $30-\mu \mathrm{m}$ resolution aerial image are shown in Fig. 1 as a function of percent scale change $a$ and rotational angle $\theta_{0}$ between the input and the object used to form the matched filter. A dramatic and disastrous loss in SNR from 30 to $3 \mathrm{~dB}$ occurs for a small 2 percent scale change or a $3.5^{\circ}$ rotation. Accurate translational positioning (within $2.5 \mu \mathrm{m}$ for a very moderate resolution input) of the matched spatial filter is also required [14] to avoid a large loss in SNR. These practical problems (caused by the reliance on the optical Fourier transform) have limited the realization of optical correlators.

Various solutions to these problems have been advanced. Multiple spatial filters of the object to be recognized at different scale changes and rotational angles can be synthesized and placed in the transform plane. However, the sophisticated synthesis system required for such a matched-filter bank, the loss in diffraction efficiency (proportional to the square of the number of multiply coded filters) and the high-resolution detector required, limit the practicality of such an approach.

Manuscript received February 27, 1976. This work was supported by the Office of Naval Research under Contract NR 350-011 and supported in part by the Air Force Office of Scientific Research, Air Force Systems Command, under Grant AFOSR-75-2851.

The authors are with the Department of Electrical Engineering, Carnegie-Mellon University, Pittsburgh, PA 15213.

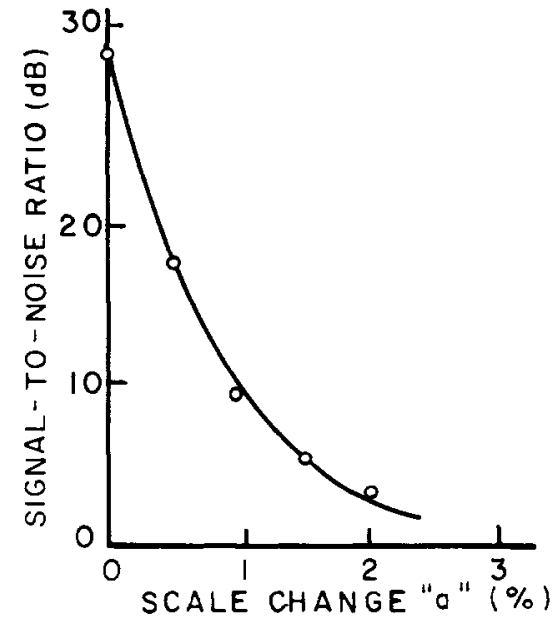

(a)

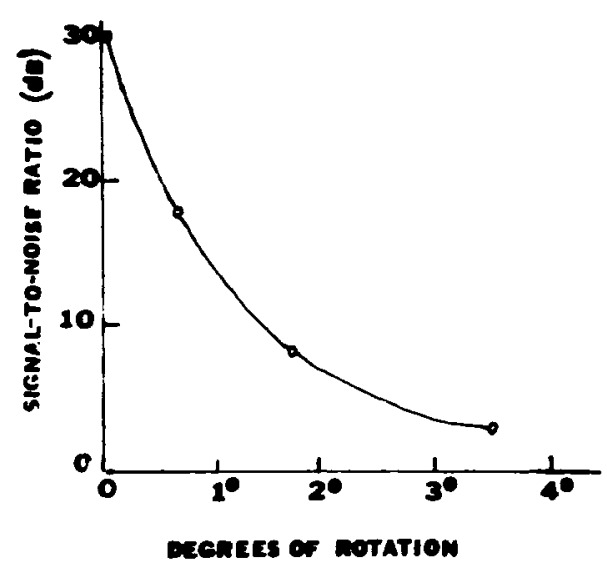

(b)

Fig. 1. (a) SNR of the correlation peak for an aerial image versus the percent scale change between the input and the reference function used to produce the matched spatial filter. (b) SNR of the correlation peak for an aerial image versus the rotation $\theta_{0}$ between the input and the reference function.

One solution to the rotational and orientation problem is to include a mechanical positioning mechanism on the input or filter to "walk on" the system into alignment. The mechanical movement of components is a severe disadvantage of this scheme in real-time processing. With the input object placed behind the transform lens, the scale of the transform can be changed [15] by moving the input and transform lens, and small scale changes of 20 percent compensated [16]. However this scheme again requires mechanical movement of components and thus does not enable realization of the real-time processing potential of an optical computer. Other approaches, such as the use of averaged filters [14], pose other practical problems.

A novel approach to these problems is considered in this paper. Rather than using conventional Fourier transforms 
which are neither scale nor rotational invariant, new optical transformations are considered. A scale-invariant optical Mellin transform is considered first. A combined FourierMellin transform, with the positional and scale invariance required for correlation, is then discussed. By combining geometrical transformations and these prior operations, a positional, rotational, and scale invariant (PRSI) transformation can be realized. The optical realization of these new transformations in real time using operational amplifier modules and computer-generated masks are considered, and initial experimental results presented.

\section{Mellin Transforms}

The Mellin transform [17] arises in the analysis of timevarying networks [18] in space-variant image restoration [19], and in the analysis of linear shift-variant imaging systems [20]. Its use in speech processing and its digital implementation have also been considered [21]. The two-dimensional Mellin transform $M(j u, j v)$ of a function $f(x, y)$ along the imaginary axis is defined by [17].

$$
M(j u, j v)=\int_{0}^{\infty} f(x, y) x^{-j u-1} y^{-j v-1} d x d y
$$

The Mellin transforms of several simple functions [18] are given in Table I.

The scale invariance [22] of the Mellin transform is of particular importance in pattern recognition. To demonstrate this scale invariance [21], consider a simple rectangular input function extending from $x_{1}$ to $x_{2}$ and $y_{1}$ to $y_{2}$,

$$
f(x, y)=\operatorname{Rect}\left[\frac{x-\left(x_{1}+x_{2}\right) / 2}{x_{2}-x_{1}}\right] \operatorname{Rect}\left[\frac{y-\left(y_{1}+y_{2}\right) / 2}{y_{2}-y_{1}}\right] \text {. }
$$

The magnitude of its Mellin transform

$$
|M(j u, j v)|=\left|\frac{4}{u v} \sin \left(u \ln \frac{x_{2}}{x_{1}}\right) \sin \left(v \ln \frac{y_{2}}{y_{1}}\right)\right|
$$

depends only on the ratios $x_{2} / x_{1}$ and $y_{2} / y_{1}$ and is thus invariant to a scale change in the input. If $M_{1}(j u, j u)$ is the Mellin transform of $f_{1}(x, y)$ and $M_{2}(j u, j v)$ is the Mellin transform of $f_{2}(x, y)=f(a x, a y)$, then

$$
M_{2}(j u, j v)=a^{-j u-j v} M_{1}(j u, j v)
$$

and $\left|M_{2}\right|=\left|M_{1}\right|$. For simplicity, only one-dimensional functions will be considered and the Mellin transform of $f(x)$ will be denoted by $M(u)$.

\section{A. Real-Time Optical Realization}

Of particular importance in the optical realization of a Mellin transform is its implementation in terms of the Fourier transform. Fortunately, the Mellin transform of $f(x)$ is the Fourier transform of $f(\exp \xi)$ [20]

$$
M(u)=\int_{-\infty}^{\infty} f(\exp \xi) \exp (-j u \xi) d \xi
$$

The Mellin transform can thus be realized by logarithmically scaling the coordinates of the input function and Fourier
TABLE I

Mellin Transforms $M(j u)$ of Several Simple Functions $f(x)$ [18]

\begin{tabular}{ll}
\hline \multicolumn{1}{c}{$f(x)$} & \multicolumn{1}{c}{$M(j u)$} \\
\hline$\delta(x-1)$ (impulse) & 1 \\
$u(x-1)$ (unit step) & $-1 / j u$ \\
$x$ & $-1 /(j u+1)$ \\
$x^{-a}$ & $-1 /(j u-a)$ \\
$x \ln x$ & $1 /(j u+1)^{2}$ \\
$e^{-a x}$ & $a^{-j u} \Gamma(j u)$ \\
$e^{-x^{2}}$ & $(1 / 2) \Gamma(j u / 2)$ \\
$\sin x$ & $\Gamma(j u) \sin (\pi j u / 2)$ \\
$\cos x$ & $\Gamma(j u) \cos (\pi j u / 2)$ \\
\hline
\end{tabular}

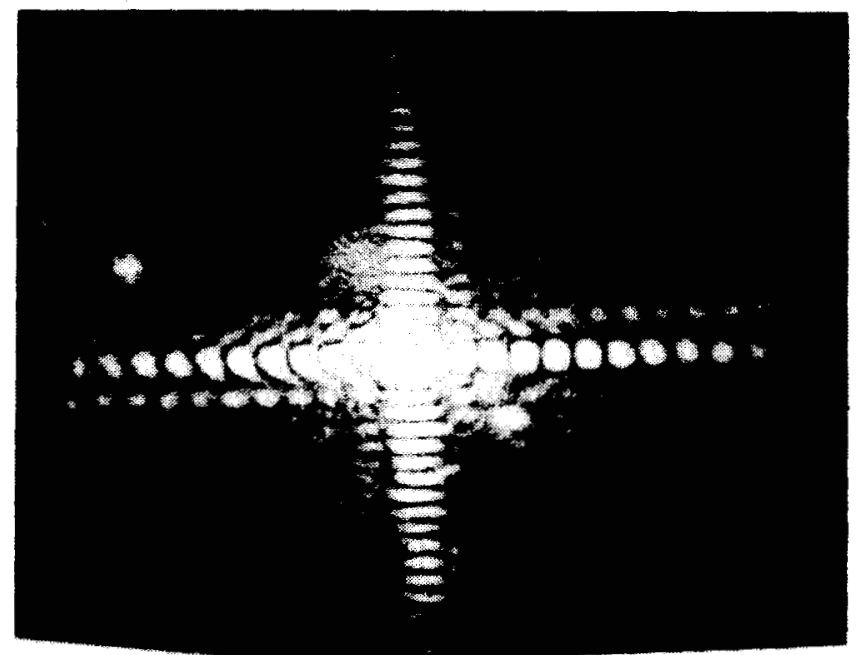

(a)

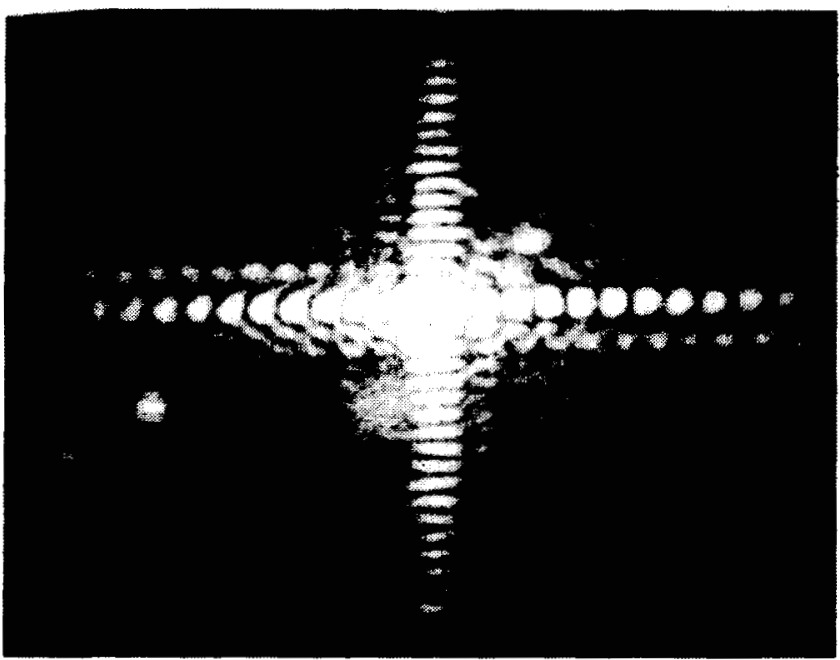

(b)

Fig. 2. Real-time optically produced Mellin transform of an input square. (a) At a width of $w$. (b) At a width of $2 w$.

transforming the resultant scaled functions. Full realization of the potential of an optical processor occurs when realtime reusable spatial light modulators are used [13]. Whether these devices are addressed by a scanning laser beam or a scan- 
ning electron beam, a transparent version of the scaled function $f(\exp \xi, \exp \eta)$ can be realized in real time from the $f(x, y)$ input by $\log$ modules in the deflection system of the input transfer. The optical Fourier transform and hence, the optical Mellin transform of $f(x, y)$ results in real time [23].

As an initial demonstration of the scale invariance of the Mellin transform and its real-time optical realization, transparencies of various simple input functions differing in scale by 200 percent to 400 percent were prepared. A closedcircuit camera was focused on these inputs. The horizontal and vertical driver outputs of the camera were level-shifted, amplified, and passed through $\log$ modules to a $Z$-modulated CRT. Transparencies of the $f(\exp \xi$, exp $\eta)$ pattern on the CRT were prepared and their transform obtained optically. The resultant Mellin transforms of two square input functions that differ in scale by 200 percent are shown in Fig. 2. The identical lens system was used in both cases and as predicted the transforms are invariant to the scale of the input.

\section{B. Coordinate Transformation Using Computer Generated Holograms}

The required $x=\exp \xi, y=\exp \eta$ coordinate conversion has been also produced by computer generated masks. The input $f(x, y)$ is placed in contact with the appropriate mask in the front focal plane of a transform lens and illuminated with laser light. The light distribution in the back focal plane of the lens will be the desired $f(\exp \xi$, $\exp \eta)$ coordinate transform of $f(x, y)$, if the computer generated mask is chosen appropriately. The use of computer-generated holograms to perform coordinate transformations has been discussed [1]. If the input $f(x, y)$ is real, the mask is a phase function $\exp [j \phi(x, y)]$ and the Fourier transform of the input and mask is

$$
\begin{aligned}
F\left(\omega_{x}, \omega_{y}\right)= & \iint f(x, y) \exp [j \phi(x, y)] \\
& \cdot \exp \left[-j\left(\omega_{x} x+\omega_{y} y\right)\right] d x d y
\end{aligned}
$$

Using the approximate saddle-point-integration method [24], the major contribution to the integral in (6) occurs at $\left(x_{0}, y_{0}\right)$, where

$$
\left.\frac{\partial \phi_{0}(x, y)}{\partial x}\right|_{x_{0}, y_{0}}=\left.\omega_{x} \quad \frac{\partial \phi(x, y)}{\partial y}\right|_{x_{0}, y_{0}}=\omega_{y} .
$$

To perform the desired coordinate transformation $\omega_{x} \propto \ln x$, $\omega_{y} \propto \ln y$, set

$$
\frac{\partial \phi(x, y)}{\partial x}=\ln x \quad \frac{\partial \phi(x, y)}{\partial y}=\ln y
$$

from which one obtains

$$
\phi(x, y)=x \ln x-x+y \ln y-y .
$$

The contribution from each saddle point is [1]

$$
\left|F\left(\omega_{x_{0}}, \omega_{y_{0}}\right)\right|^{2}=\left|4 \pi^{2} f^{2}\left(x_{0}, y_{0}\right) /\left(\phi_{x x} \phi_{y y}-\phi_{x y}^{2}\right)\right|
$$

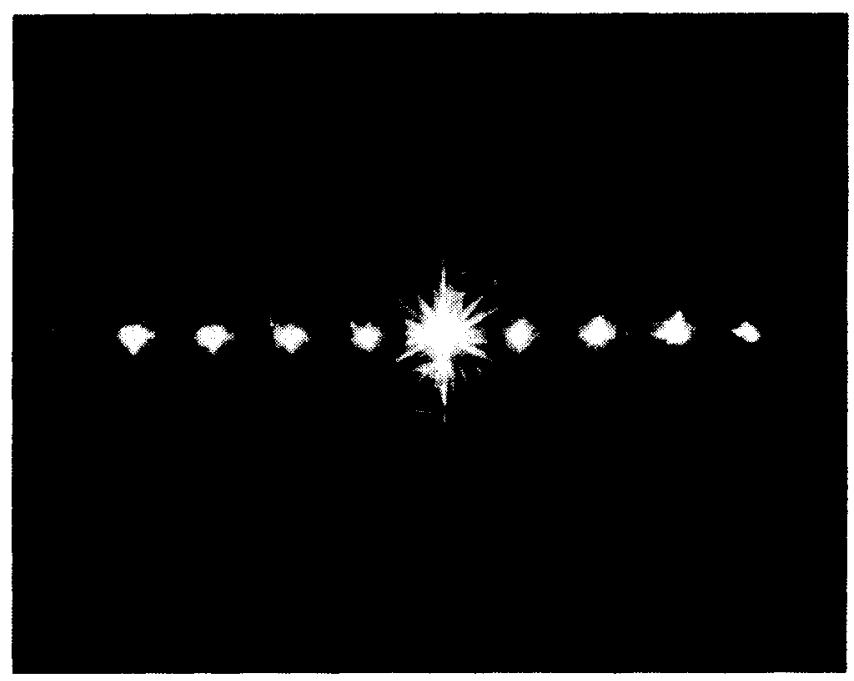

Fig. 3. Fourier transform of a computer-generated mask to produce the logarithmic coordinate scaling using a circular input object.

where $\phi_{x x}=\partial^{2} \phi / \partial x^{2}=1 / x, \phi_{y y}=\partial^{2} \phi / \partial y^{2}=1 / y$, and $\phi_{x y}=$ $\partial^{2} \phi / \partial x \partial y=0$.

A computer-generated logarithmic transformation mask was fabricated using the method described by Lee [25]. In this method, the hologram fringes are constructed to satisfy $\cos [\phi(x, y)]>C$, where $C$ is a constant between zero and one. When photoreduced by twenty, to $2 \times 2 \mathrm{~cm}$, the resultant mask resolution was 9 lines/mm (higher resolution of 100 lines $/ \mathrm{mm}$ is possible with improved plotter routines). The Fourier transform of the computer generated mask for a logarithmic coordinate transformation is shown in Fig. 3 for the case of a circular input function. The first four harmonics of the transformed circle are shown.

While these preliminary results appear promising, this technique as applied to Mellin transforms requires improvement. Since the scaled input is not formed on a parallel beam, it must be recorded before further processing is possible. In addition, the amplitude weighting proportional to $x y$ in (10) must also be removed to prevent shading effects. At present the low hologram-mask resolution prevents the use of high-resolution input functions. This scheme is also only applicable to conformal mapping transformations and is thus not useful in the polar transformation to be discussed in Section IV.

\section{Digital Mellin Transform}

The Mellin transform can also be implemented by integration according to (1). Such a direct implementation is possible by digital processing. In this case, the integration is replaced by a summation, and the one-dimensional version of (1) becomes

$$
M(k \Delta u)=\sum_{i=1}^{N} f(i \Delta x)(i \Delta x)^{j k \Delta u-1} \Delta x
$$

where the input and transform space resolutions are $\Delta x$ and $\Delta u$ respectively. The two-dimensional $N \times M$ Mellin transform can be realized by matrix multiplication as $M=T f T^{t}$, where $f$ is an $N \times N$ matrix of the sampled input, $T$ is the 


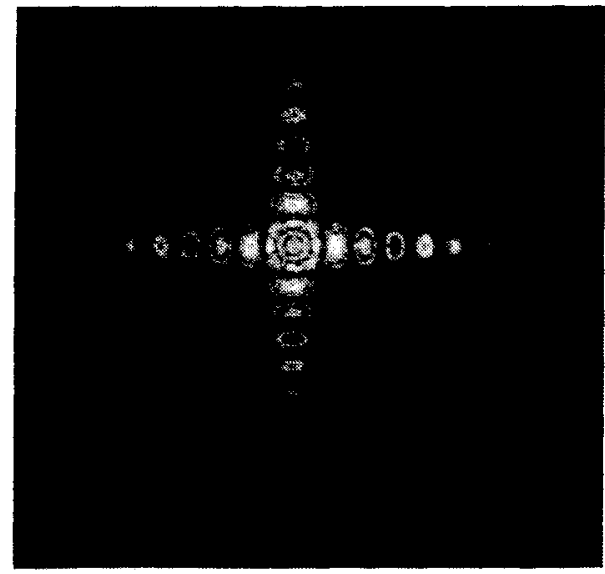

(a)

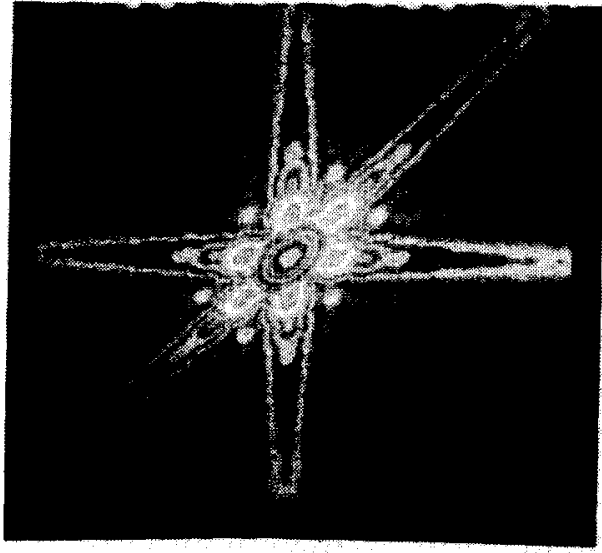

(b)

Fig. 4. Digitally computed Mellin transforms. (a) For square input objects. (b) For triangular input objects.

transformation matrix and $T^{t}$ is the transposed matrix. It can be shown [21] that a scale and shift invariant transform results if the Mellin transform is applied to the magnitude of the Fourier transform

$$
T=\frac{1}{\Delta x}\left[\begin{array}{ccc}
\exp (j \Delta u \ln \Delta x) \frac{1}{2} \exp (j \Delta u \ln 2 \Delta x) & \cdots \frac{1}{N} \exp (j \Delta u \ln N \Delta x) \\
\vdots & \vdots \\
\exp (j M \Delta u \ln \Delta x) \frac{1}{2} \exp (j M \Delta u \ln 2 \Delta x) & \cdots \frac{1}{N} \exp (j M \Delta u \ln N \Delta x)
\end{array}\right]
$$

Since no fast computational algorithm is yet available for implementing $M=T f T^{t}$, the processing time required for a digital implementation can be quite long. However, a digital Mellin transform can also be realized by exponentially sampling the input and performing a two-dimensional FFT on this data. The digital Mellin transforms for several simple inputs are shown [21] in Fig. 5. The input sample size $(256 \times 256)$ determined the sampling rate used. These calculations were repeated for these inputs with a 200-percent scale change and the resultant outputs were identical.

\section{SCALE-INVARIANt CoRrelation}

For this discussion let us consider two one-dimensional functions $f_{1}(x)$ and $f_{2}(x)$ that differ only in scale. We will use the following notation:

$$
\begin{array}{ll}
f_{1}(x)=f(x), f_{2}(x)=f(a x): & \text { Two input functions } \\
F_{1}\left(\omega_{x}\right), F_{2}\left(\omega_{x}\right): & \text { The Fourier transforms of } \\
& f_{1} \text { and } f_{2} \\
M_{1}(u), M_{2}(u): & \begin{array}{l}
\text { The Mellin transforms of } \\
f_{1} \text { and } f_{2}
\end{array} \\
M_{1}^{\prime}\left(u^{\prime}\right), M_{2}^{\prime}\left(u^{\prime}\right): & \text { The Mellin transforms of } \\
& \left|F_{1}\right| \text { and }\left|F_{2}\right| .
\end{array}
$$

The basis for conventional optical correlation using matched spatial filters [12] is the shift invariance of the Fourier transform. The magnitude of the Fourier transform of an object or function is invariant to a shift in the function $|F\{f(x)\}|=$ $\left|F\left\{f\left(x-x_{0}\right)\right\}\right|$ but not to a scale change in the input. Conversely, the magnitude of the Mellin transform is invariant to a scale change in the input [see (4)], but not a shift in the input. Since a scale change in the input scales the magnitude of its Fourier transform directly, $F\{f(a x)\}=(1 /|a|) F\left(\omega_{x} / a\right)$,
[23], [26]. This scale and shift invariance is necessary in practice, when the input data are not scaled about the geometric center of the input aperture.

The Mellin-type correlation of two functions $f_{1}$ and $f_{2}$ can be defined as,

$$
f_{1}(x) \otimes f_{2}(x)=\int_{0}^{\infty} f_{1}(y) f_{2}^{*}(x y)(1 / y) d y
$$

where $\otimes$ denotes a Mellin-type correlation and $*$ denotes the complex conjugate. By substituting $x=\exp \xi, y=\exp \eta$ into equation (13), the conventional correlation $f_{1}(\exp \xi) \otimes f_{2}(\exp \xi)$ results. An inverse Mellin transform $M^{-1}$ can also be defined,

$$
M^{-1}\{M(u)\}=f(x)=\frac{1}{2} \pi \int_{-\infty}^{\infty} M(u) x^{j u} d u .
$$

Using the definitions in (1), (5), (13), and (14), the above notation, and basic linear-system theory, the four methods of scale invariant correlation noted in Table II can be distinguished [26].

Correlation methods (C) and (D) in Table II are preferable for optical (or digital) implementation since they use the more easily implemented Fourier transform operation rather than the inverse Mellin transform (methods (A) and (B)). Method (C) results in the correlation of the input functions rather than their Fourier transforms (as in (D)); however the correlation in (D) is both scale and shift invariant, whereas that in (C) requires proper positioning of the input functions. The basic steps in the correlation by methods (C) and (D) are the same except for the added initial step in (D) in which the magnitudes of the Fourier transforms of the functions are formed. 
TABLE II

Folr Methods or Scale Invariant Correlation [26]. $M$ Denotes the Mellis Traishorm, $F$ the Folrier Transhorm, (OMElin-Type Correlation, and $\odot$ Convfitional Correlation
(A) $M^{-1}\left\{M_{1} M_{2}^{*}\right\}=f_{1}(x) \otimes f_{2}(x)$
(B) $M^{-1}\left\{M_{1}^{\prime} M_{2}^{*}\right\}=\left|F_{1}\left(\omega_{x}\right)\right| \otimes\left|F_{2}\left(\omega_{x}\right)\right|$
(C) $F\left\{M_{1} M_{2}^{*}\right\}=f_{1}(\exp x) \odot f_{2}(\exp x)$
(D) $F\left\{M_{1}^{\prime} M_{2}^{*}\right\}=\left|F_{1}\left(\exp \omega_{x}\right)\right| \odot\left|F_{2}\left(\exp \omega_{x}\right)\right|$

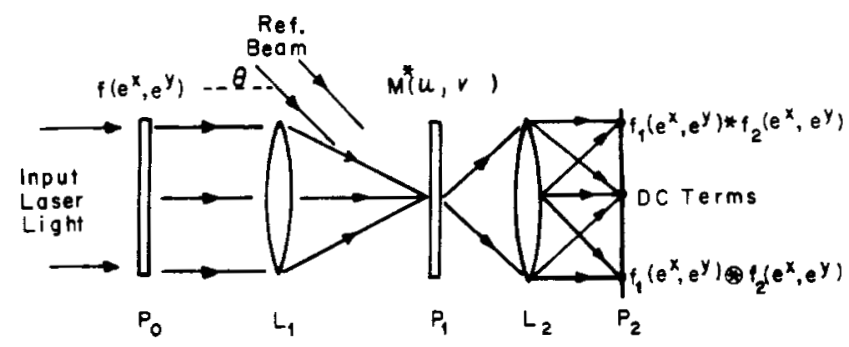

Fig. 5. Schematic diagram of an optical scale invariant correlator.

As an initial demonstration of optical scale-invariant correlation, the optical system in Fig. 5 was used to implement method (C). The scaled function $f_{2}(\exp \xi$, $\exp \eta)$ was produced from $f_{2}(a x, a y)$ (in real time by log modules in the deflection system as described in Section II-A) and placed at plane $P_{0}$ in Fig. 5. The Fourier transform of the transmittance of $P_{0}$ is formed at $P_{1}$ by lens $L_{1}$. When this transform is interfered with an off-axis plane-wave reference beam at an angle $\theta$, the pattern recorded on an intensity sensitive material (e.g., film) at $P_{1}$ consists of four terms (as in conventional holographic spatial filter synthesis [12]). One of these terms is proportional to $M_{2}^{*}$. With the reference beam blocked and the transmittance of $P_{0}$ proportional to $f_{2}$, the autocorrelation will occur at $x_{2}^{\prime}=0, y_{2}^{\prime}=-f \sin \theta$ in plane $P_{2}$. With $f_{1}$ placed at $P_{0}$ and the same Mellin hologram $M_{2}^{*}$ at $P_{1}$, plane $P_{2}$ will contain the cross-correlation of $f_{1}$ and $f_{2}$. The coordinates of the cross-correlation will be shifted [27] to $x_{2}^{\prime}=K \ln a, y_{2}^{\prime}=-f \sin \theta+K \ln a$, where $K$ is a constant that depends on the magnification of the optical system, $a$ is the scale factor between the inputs, $\theta$ is the reference beam angie, and $f$ is the focal length of the transform lenses $L_{1}$ and $L_{2}$.

Two squares differing in scale by 200 percent were used as the inputs $f_{1}(x, y)$ and $f_{2}(x, y)$ in these initial optical demonstrations. The Mellin transform hologram $M_{2}^{*}$ of the smaller square was formed and stored at $P_{1}$. With the logarithmically scaled function $f_{2}(\exp \xi$, $\exp \eta)$ placed at $P_{0}$, the autocorrelation of $f_{2}(\exp \xi$, exp $\eta)$ occurs at $P_{2}$, whereas with the logarithmically scaled larger square placed at $P_{0}$, the crosscorrelation of the two squares occurs at $P_{2}$. The optical crosscorrelation peak, obtained in real time, is shown in Fig. 6(a). Cross-sectional scans (obtained with a scanning photometric microscope) of the autocorrelation and cross-correlation peaks are shown in Fig. 6(b).

As shown by Fig. 6(a), it is possible to correlate two objects that differ in scale by 200 percent. As the cross-sectional plots in Fig. 6(b) demonstrate, there is no loss in the SNR of the correlation peak, regardless of whether both smaller squares are correlated (autocorrelation) or the smaller square is correlated with the larger square (cross-correlation). Finally, the location of the cross-correlation peak is shifted from the onaxis position of the autocorrelation peak by an amount pro-

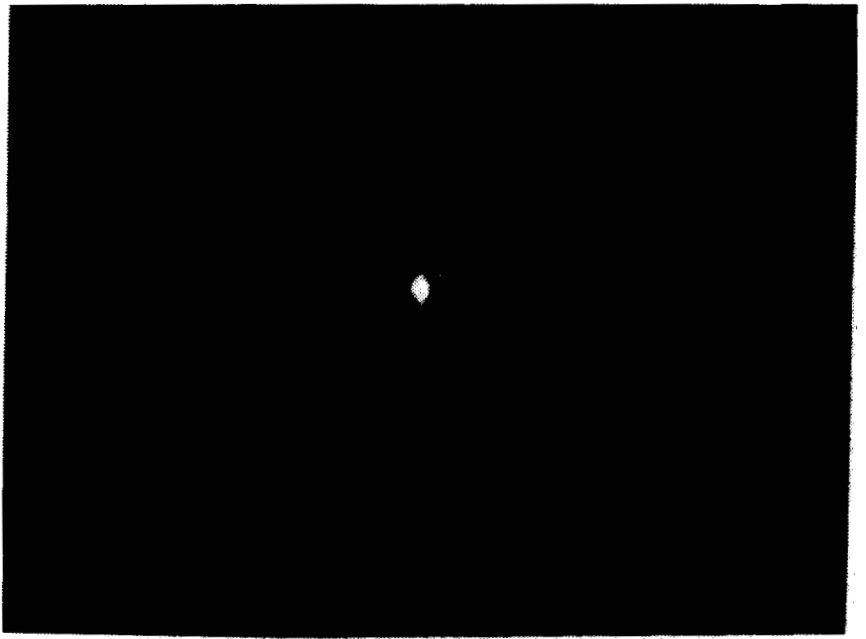

(a)

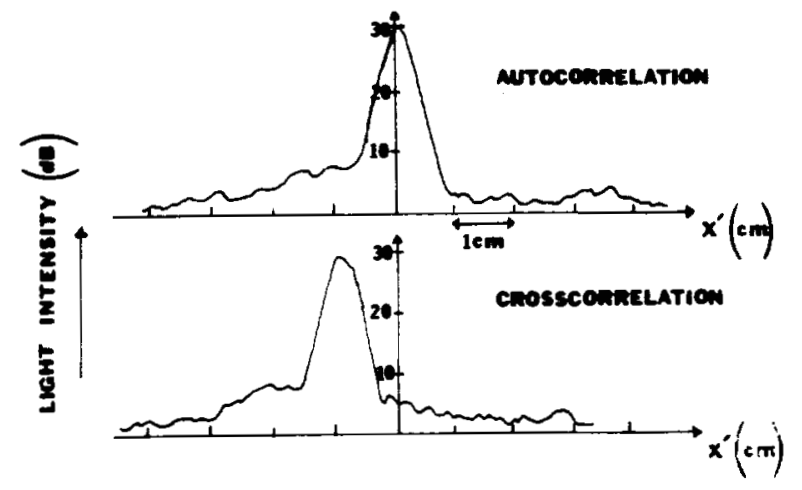

(b)

Fig. 6. Scale invariant optical correlation of two square input functions that differ in scale by 200 percent. (a) Cross-correlation peak. (b) Cross-section of the autocorrelation and cross-correlation peaks.

portional to the scale change between the inputs. From the location of the correlation peak, the scale change can thus be determined and appropriate steps taken depending on the application. The use of computer-generated masks rather than log modules to effect the initial scaling of the inputs is possible if higher resolution masks can be fabricated.

\section{Positional, Rotational, and Scale INVARIANT CORRELATION}

By properly combining the Fourier-Mellin transform with a polar transformation on the input data, a PRSI transformation can be realized [27]. Consider two functions $f_{1}$ and $f_{2}$ to be correlated, where $f_{2}$ is a scaled and rotated version of $f_{1}$ with a scaling factor $a$ and rotational angle $\theta$ between the input objects. The PRSI transformation can be realized by the following steps:

1) the magnitudes $\left|F_{1}\left(\omega_{x}, \omega_{y}\right)\right|$ and $\left|F_{2}\left(\omega_{x}, \omega_{y}\right)\right|$ of the Fourier transforms of the two input objects are formed;

2) these functions in step 1) are converted to polar coordinates as $F_{1}(r, \theta)$ and $F_{2}(r, \theta)$;

$3)$ these polar functions are then logarithmically scaled in $r$ to yield $F_{1}(\exp \rho, \theta)$ and $F_{2}(\exp \rho, \theta)$

4) the Fourier transforms of the functions in step 3) yield the PRSI functions $M_{1}\left(\omega_{\rho}, \omega_{\theta}\right)$ and $M_{2}\left(\omega_{\rho}, \omega_{\theta}\right)$. 


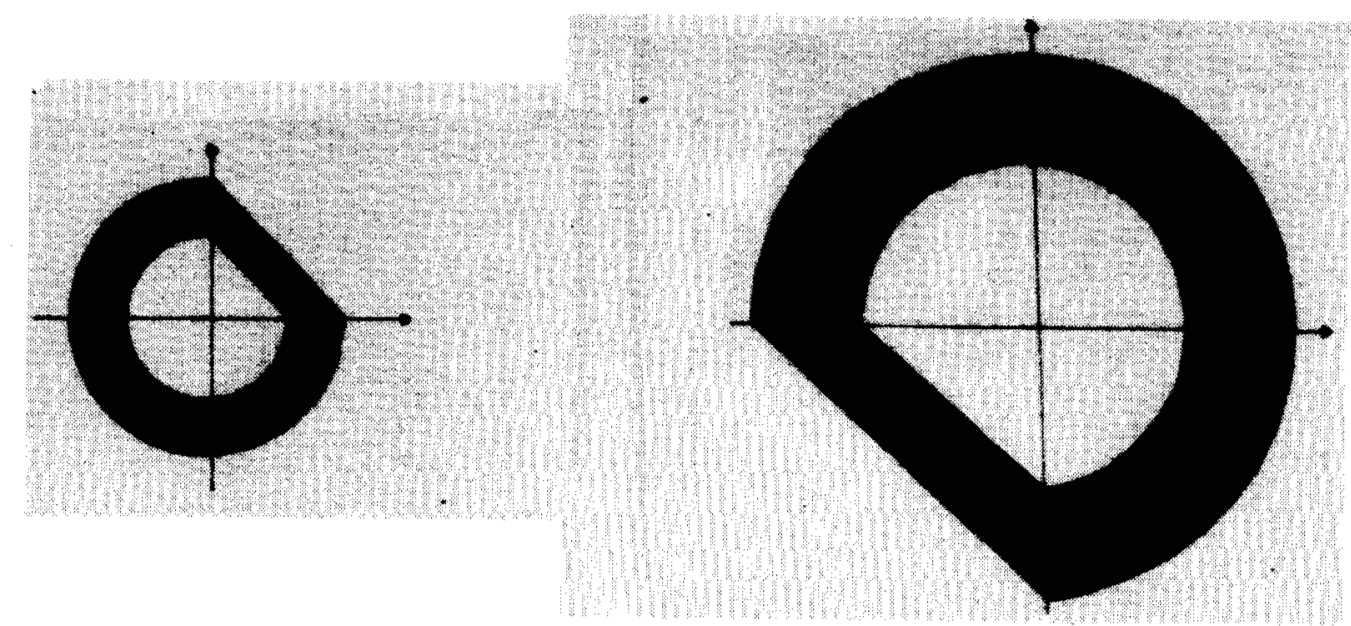

(a)

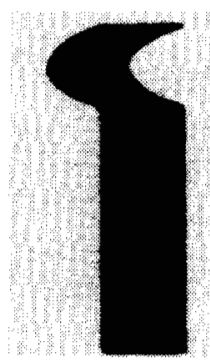

(c)

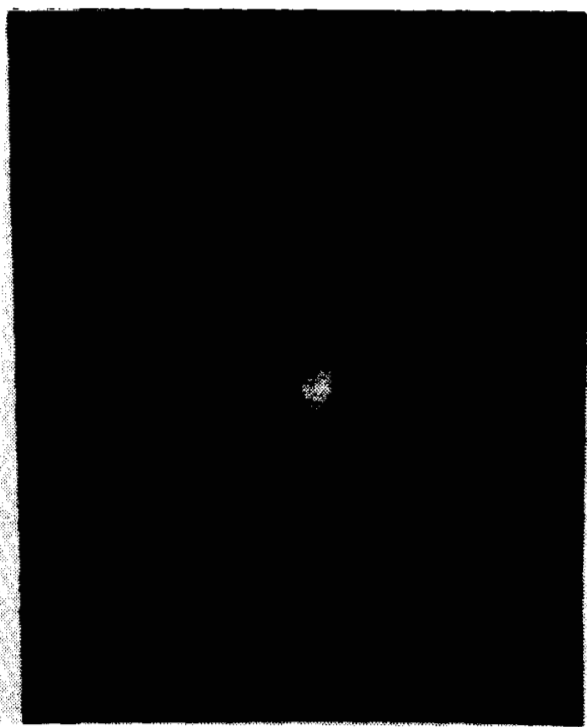

(e) (b)

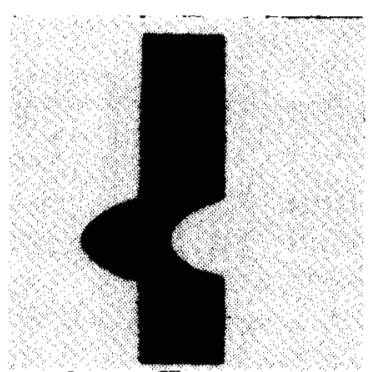

(d)

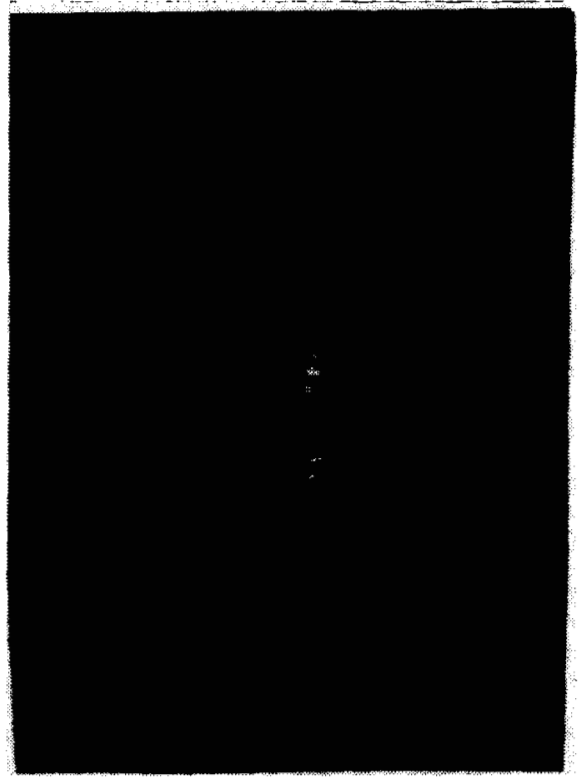

(f)

Fig. 7. Rotational and scale infariant optical correlation of two input functions that differ in scale by 200 percent and in rotation by $180^{\circ}$. (a) and (b) Inputs. (c) and (d) Polar transformed and logarithmically acaled functions. (e) Autocorrelation of (a). (f) Cross-correlation of both inputs.

Steps 1) and 4) can be realized optically by a Fourier transform lens. Steps 2) and 3) can be performed with operational amplifier-modules computer-generated masks or other methods. Steps 2) and 3) can be combined into one, and are separated only for analy sis purposes. The final Fourier transform in step 4 ) in the logarithmically scaled $r$ coordinate direction is equivalent to a Mellin transform, and thus the final PRSI functions are denoted as $M_{1}$ and $M_{2}$.

The invariance of this transformation follows from an analysis of the synthesis steps [27]. The positional variations in the input functions are removed in step 1) because of the basic shift invariance of the magnitude of the Fourier transform. The polar transformation in step 2) separates the effects of scale changes and rotations between the two inputs. Scale variations between the inputs will scale the $r$ coordinate directly, while a rotation between inputs by $\theta_{0}$ will appear only as a shift in the $\theta$ coordinate. Steps 3) and 4) are equivalent to a Mellin transform in $r$. Since scale variations affect 
only the $r$ coordinate and since the Mellin transform in $r$ is scale invariant, the resultant functions will be scale invariant. The combination of the conversion of a rotational difference to a shift by step 2) and the shift invariance of the Fourier transform in step 4) result in a final function that is rotationally invariant.

All required operations in the PRSI transformation can be realized by the methods previously noted. However, in this case it has not yet been possible to use computer-generated masks to effect the polar coordinate transformation since the required functions are not separable. Future developments may make such operations possible.

Since a detailed analysis of these steps is available in the literature [27], this present discussion will concentrate on the initial optical realization of this PRSI transformation and the correlation of two objects that differ in both scale and rotation. Before describing the experiments performed, one aspect of the effects of rotation must be discussed. While a rotation in the input does produce only a shift in the $\theta$ coordinate of the polar function, the shift is not the same for all parts of the functions. To visualize this, denote the portion of $F_{1}\left(\omega_{x}, \omega_{y}\right)$ that extends from 0 to $-\theta_{0}$ by $F_{1 a}$ and the remaining portion from 0 to $2 \pi-\theta_{0}$ by $F_{1 b}$. The version of $F_{1}$ rotated by $\theta_{0}$ and denoted by $F_{2}$ has two components also.

Comparing $F_{1}(r, \theta)$ and $F_{2}(r, \theta)$, the region of $F_{2}(r, \theta)$ corresponding to $F_{1 a}(r, \theta)$ is found to be shifted upward by $\theta_{0}$ while the region of $F_{2}(r, \theta)$ associated with $F_{1 b}(r, \theta)$ is shifted down by $2 \pi-\theta_{0}$ in the plot of $F_{2}(r, \theta)$. The final PRSI function $M_{2}$ is thus related to the two components $M_{1 a}$ and $M_{1 b}$ of the first PRSI function by

$$
\begin{aligned}
M_{2}\left(\omega_{\rho}, \omega_{\theta}\right)= & M_{1 a}\left(\omega_{\rho}, \omega_{\theta}\right) \\
& \cdot \exp \left[-j\left(\omega_{\rho} \ln a+\omega_{\theta} \theta_{0}\right)\right]+M_{1 b}\left(\omega_{\rho}, \omega_{\theta}\right) \\
& \cdot \exp \left\{-j\left(\omega_{\rho} \ln a+\omega_{0}\left(2 \pi-\theta_{0}\right)\right]\right\} .
\end{aligned}
$$

In the correlation using PRSI functions, $M_{1}^{*}\left(\omega_{\rho}, \omega_{\theta}\right)$ will be recorded at plane $P_{1}$ in a system like that of Fig. 5 and $F_{2}(\exp \rho, \theta)$ placed at $P_{0}$. The transmittance of $P_{1}$ will then be proportional to $M_{1}^{*} M_{2}$ and the output plane $P_{2}$ will contain the correlation of $F_{1}$ and $F_{2}$. However, the output will now contain two correlation peaks: a) the cross-correlation $F_{1 a} \circledast F_{2}$ at $\rho^{\prime}=\ln a$ and $\theta^{\prime}=\theta_{0}$ and; b) the cross-correlation $F_{1 b} \odot F_{2}$ at $\rho^{\prime}=\ln a$ and $\theta^{\prime}=-2 \pi+\theta_{0}$; where the coordinates of $P_{2}$ are $\left(\rho^{\prime}, \theta^{\prime}\right)$. The intensities of these two correlation peaks must be added if no loss in SNR is to occur between the autocorrelation of two identical input objects and the crosscorrelation of two input objects that differ in scale and rotation. This summation can be realized by a fixed pair of detectors separated by a distance corresponding to $\theta^{\prime}=2 \pi$. If the rotational information is not needed, the output plane can be scanned in the $\rho^{\prime}$ direction with a slit. A single correlation peak results, whose relative position is proportional to the log of the scale change $a$.

As an initial demonstration of scale and rotational invariant correlation, the Fourier transforms [Figs. 7(a) and 7(b)] of two input objects that differ in scale by 200 percent and in rotation by $180^{\circ}$ were formed. The polar transformed functions logarithmically scaled in $r$ were then computed [Figs. $7(\mathrm{c})$ and $7(\mathrm{~d})]$. The Mellin hologram $M_{i}^{*}\left(\omega_{\rho}, \omega_{\theta}\right)$ of the input in Fig. 7(a) was then formed at $P_{1}$ in Fig. 5 as before. With $F_{1}(\exp \rho, \theta)$ at plane $P_{0}$, the auto-correlation [Fig. $7(\mathrm{e})]$ of $F_{1}(\exp \rho, \theta)$ is formed at plane $P_{2}$. With $F_{2}(\exp \rho, \theta)$

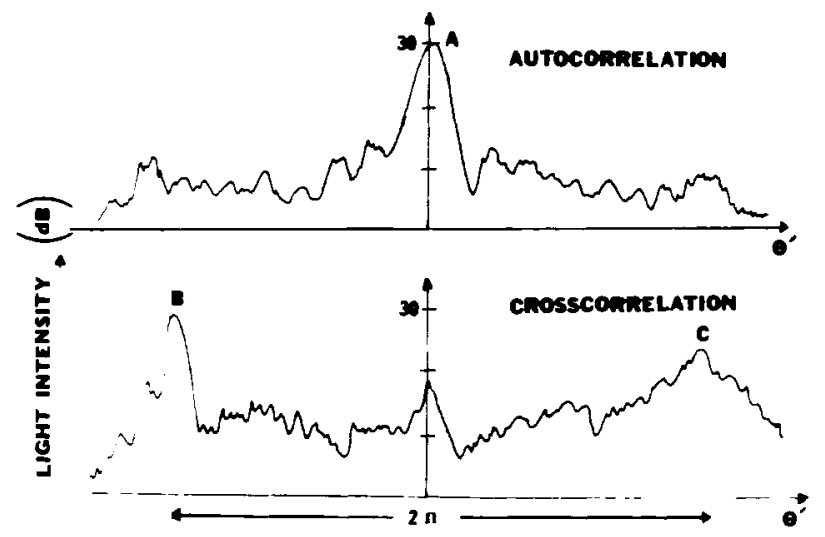

(a)

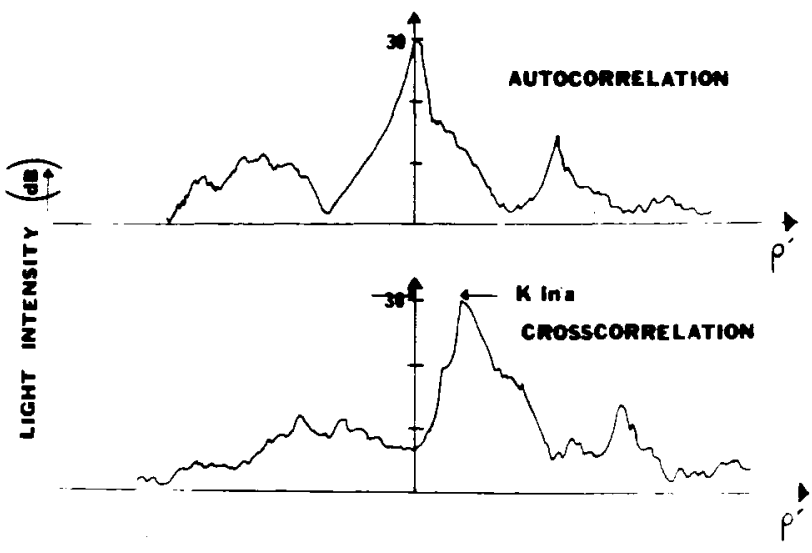

(b)

Fig. 8. Cross-sectional scans of autocorrelation and cross-correlation of input objects in Fig. 7. (a) In the $\theta^{\prime}$ direction using a point probe. (b) In the $\rho^{\prime \prime}$ direction using a slit probe.

placed at $P_{0}$ the cross-correlation [Fig. 7(f)] occurs and contains two peaks rather than one as explained above.

The cross-sectional scans [Fig. 8(a)] of the auto-correlation and correlation patterns in the $\theta^{\prime}$ direction show two crosscorrelation peaks $B$ and $C$. The sum of their intensities equals that of the auto-correlation peak $A$ so that there is no loss in SNR. These peaks are separated by $2 \pi$ and shifted from the $\theta^{\prime}$ location of the auto-correlation peak by the $\theta_{0}=\pi$ rotation between the two inputs. The cross-sectional scans [Fig. 8(b)] in the $\rho^{\prime}$ direction (using a scanning slit) show that the $\rho^{\prime}$ coordinate of the correlation is related to the scale change between the input objects and that there is again no loss in SNR between the auto-correlation and cross-correlation. While preliminary, these results show that it is possible to correlate two objects that differ in scale and rotation with no loss in SNR and that the scale and rotational differences can be found from the locations of the correlation peaks.

\section{SUMmaRY AND CONCLUSION}

A new approach to optical pattern recognition has been presented that does not rely solely on the Fourier transform operation. In theory and by experiment, it has been shown that these new operations allow objects that differ in scale and rotation to be correlated with no loss in SNR. It has also been demonstrated that the scale and rotational differences between the objects can be determined from the location of the correlation peak. 


\section{ACKNOWLEDGMENT}

The assistance of Dr. W. H. Lee of Xerox in the synthesis of the computer-generated masks, and initial technical discussions with Dr. G. Huang of Philco-Ford is gratefully recognized.

\section{REFERENCES}

[1] O. Bryngdahl, "Geometrical transformations in optics," J. Opt. Soc. Amer., vol. 64, no. 8, pp. 1092-1099, Aug. 1974.

[2] S. R. Dashiel and A. Sawchuck, "Analysis and synthesis of nonlinear optical processing," in Proc. Elec. Opt. Syst. Des. Conf., Anaheim, CA, 1975.

[3] N. Farhat, "Nonlinear optical data processing and filtering: a feasibility study," IEEE Trans. Comput., vol. C-24, pp. 443448, Apr. 1975.

[4] K. T. Stalker and S. H. Lee, "The use of nonlinear optical elements in optical information processing," J. Opt. Soc. Amer. vol. 64, p. 564, Apr. 1974.

[5] D. Jablonowski and S. Lee, "Coherent processor with optical feedback,"J. Opt. Soc. Amer., vol. 63, p. 1306, Oct. 1973.

[6] D. Casasent and W. Sterling, "An hybrid optical/digital processor: hardware and applications," IEEE Trans. Comput., vol. C-24, pp. 348-357, Apr. 1975.

[7] D. Casasent, 'Hybrid Processors," in Optical Data Processing, S. Lee, Ed. Heidelberg: Springer-Verlag, 1976.

[8] B. J. Thompson, "Hybrid processing systems-An assessment," this issue, pp. 62-76.

[9] F. Carlson, "Generalized optical operators," IEEE Intercom. Tech. Paper 7/2, Mar. 1973

[10] R. Eguchi and F. Carison, "Linear vector operations in coherent optical data processing systems," Appl. Opt., vol. 9, pp. 687694, Mar. 1970.

[11] J. W. Goodman, "Operations achievable with coherent optical information processing system," this issue, pp. 29-38.

[12] A. Van der Lugt, "Signal detection by complex spatial filtering," IEEE Trans. Inform. Theory, vol. IT-10, pp. 139-145, Apr. 1964.

[13] D. Casasent, "Spatial light modulators," this issue, pp. 00-00.

[14] S. Almeida and $K$. T. Eu, "Water polution monitoring using matched spatial filtering," Appl. Opt., Mar. 1976.

[15] J. Goodman, Introduction to Fourier Optics. New York: McGraw-Hill, 1968.

[16] Harris Semicond. Div.

[17] R. Bracewell, The Fourier Transform and Its Applicattons. New York: McGraw-Hill, 1965, ch. 12.

[18] F. R. Geradi, "Application of Mellin and Hankel transforms to networks with time varying parameters," IRE Trans. Circuit Theory, vol. CT-6, pp. 197-208, June 1959.

[19] A. Sawchuck, "Space-variant image restoration by coordinate transformations," vol. 64, pp. 138-144, Feb. 1974.

[20] G. M. Robbins and T. S. Huang, "Inverse filtering for linear shift-variant imaging systems," Proc. IEEE, vol. 60, pp. 862872 , July 1972 .

[21] G. Huang, F. Russell, and W. Chen, ELA Symposium, College Park, MD, Apr. 1975.

[22] P. Baudelaire, "Linear stretch-invariant systems," Proc. IEEE Lett., vol. 61, pp. 467-468, Apr. 1974.

[23] D. Casasent and D. Psaltis, "Scale invariant optical transform," Opt. Engr., Mar. 1976.

[24] M. Born and E. Wolf, Princtples of Optics. New York: Pergamon, 1965, p. 753

[25] W. H. Lee, "Binary synthetic holograms," Appl. Opt., vol. 13, pp. 1677-1682, July 1974 .

[26] D. Casasent and D. Psaltis, "Scale invariant optical correlation using Mellin transforms," Opt. Commun., submitted.

[27] D. Casasent and D. Psaltis, "Positioned, rotational, and scale invariant optical correlation," Appl. Opt., submitted for publication.

\title{
Laser Stereometry
}

\author{
H. J. CAULFIELD, SENIOR MEMBER, IEEE, TOMAS HIRSCHFELD, SENIOR MEMBER, IEEE, \\ J. MORRIS WEINBERG, AND R. E. HERRON
}

\begin{abstract}
Abstruct-Laser stereometry is the direct photography of unencoded three-dimensiomal object information using a lase. The particular laser stereometer described here employs range-dependent time delays in the retum of light incident on the object from a mode-locked laser pulse. Subminlimeter depth resolution over objects meters deep is achievable.
\end{abstract}

\section{INTRODUCTION}

A GROWING NEED for quantitative three-dimensional or "stereometric" information about objects in the near environment has helped to focus attention on possible improvements in stereometric-sensing technology. In this regard, much has been accomplished recently by those involved in artificial intelligence work [1] and by researchers in the new field of biostereometrics [2] -the spatial and spatiotemporal analysis of biological form and function based on principles of analytic geometry. However, it is generally acknowledged that the present armamentarium of stereometric-sensing instruments can and should be further extended.

Manuscript received June 2, 1976.

H. J. Caulfield, T. Hirschfeld, and J. M. Weinberg are with Block Engineering, Inc., Cambridge, MA 02139.

R. E. Herron is with the Texas Institute for Rehabilitation and Research, Houston, TX 77025.
The simplest type of stereometric sensor is a mechanical one derived from early surveying. In the fifteenth century, Leon Battista Alberti used an adaptation of such a device to measure and replicate a sculpture. Photooptical, electroopti$\mathrm{cal}$, and other more exotic stereometric sensors also have a long history which dates back to the apparatus used by ancient astronomers.

A "recent" method (which originated in the Mid-Nineteenth Century) is based on close-range stereophotogrammetry. Though oft maligned in recent years as being too slow, too costly, too complicated, or too something else, it endures for the simple reason that it works for a wide range of objects under a wide variety of environmental conditions. Some progress has been made towards automating the stereo-image analysis procedures but a fully satisfactory solution has yet to be found. In the meantime, semiautomatic methods of stereophotogrammetry continue to be used extensively and, apparently, quite effectively.

In the last twenty years, various interferometric methods, mainly those bảsed on holography and moire interferometry, have shown promise for stereometric sensing purposes [2] but practical problems have limited their usefulness for quantifying large dynamic objects such as a human body form. 


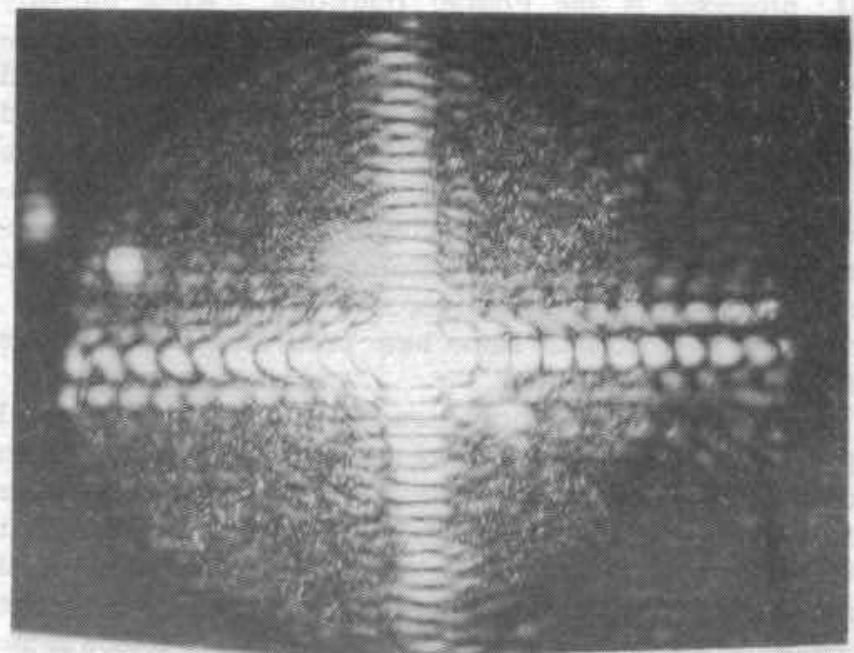

(a)

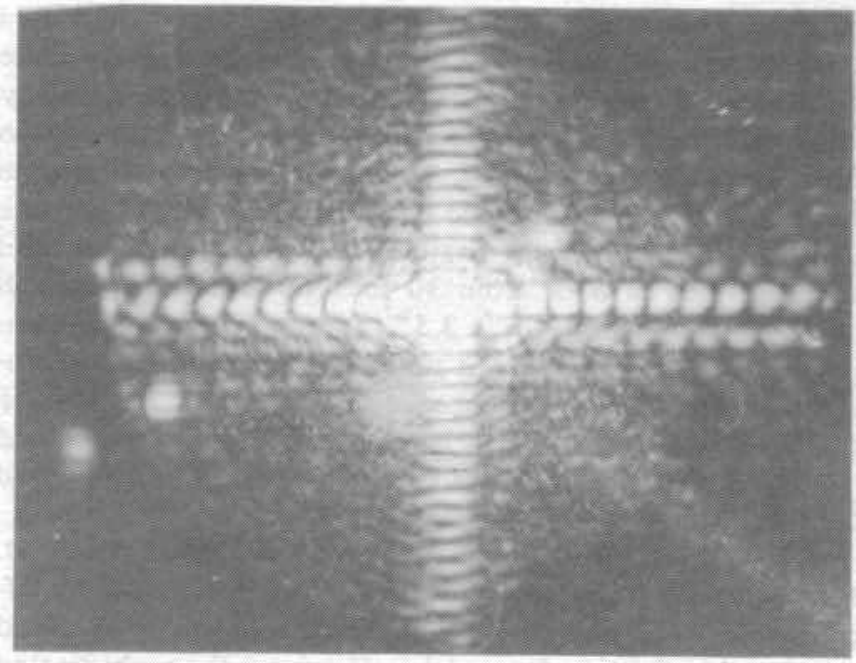

(b)

Fig. 2. Real-time optically produced Mellin transform of an input square. (a) At a width of $w$. (b) At a width of $2 w$. 


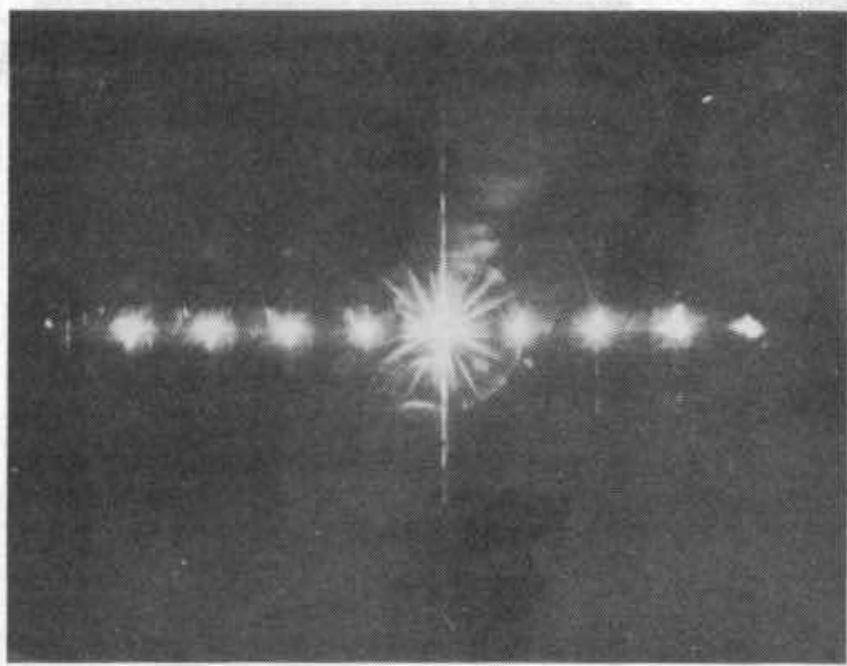

Fig. 3. Fourier transform of a computer-generated mask to produc the logarithmic coordinate scaling using a circular input object. 


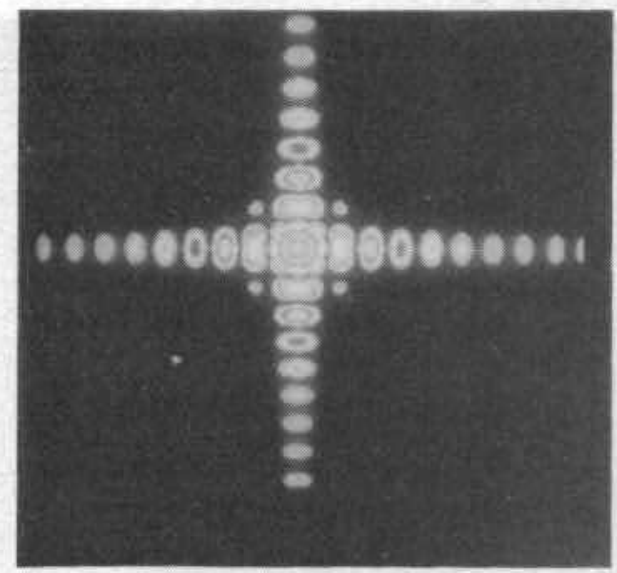

(a)

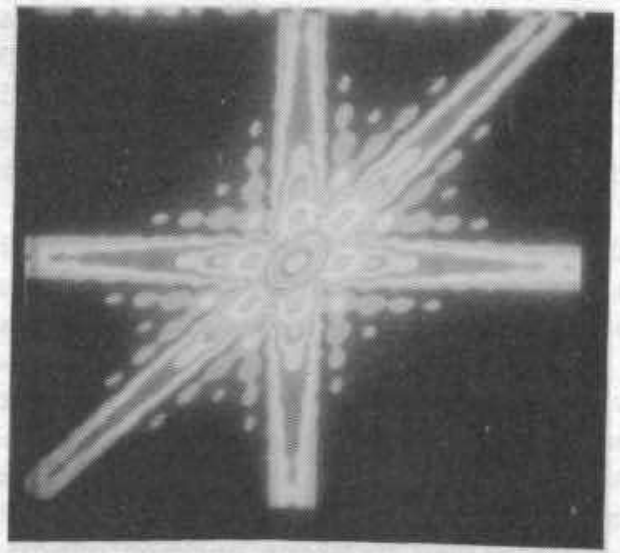

(b)

Fig. 4. Digitally computed Mellin transforms. (a) For square input objects. (b) For triangular input objects. 


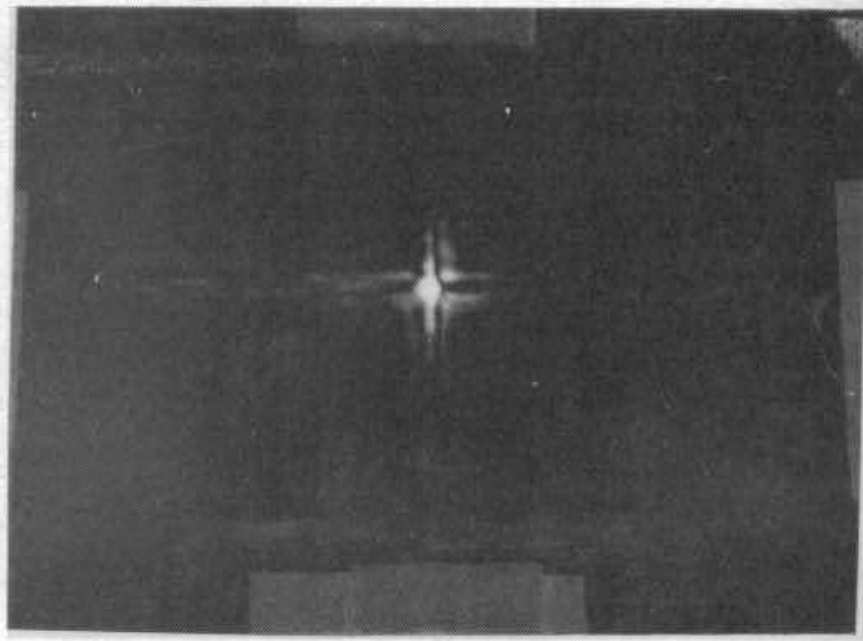

(a) 


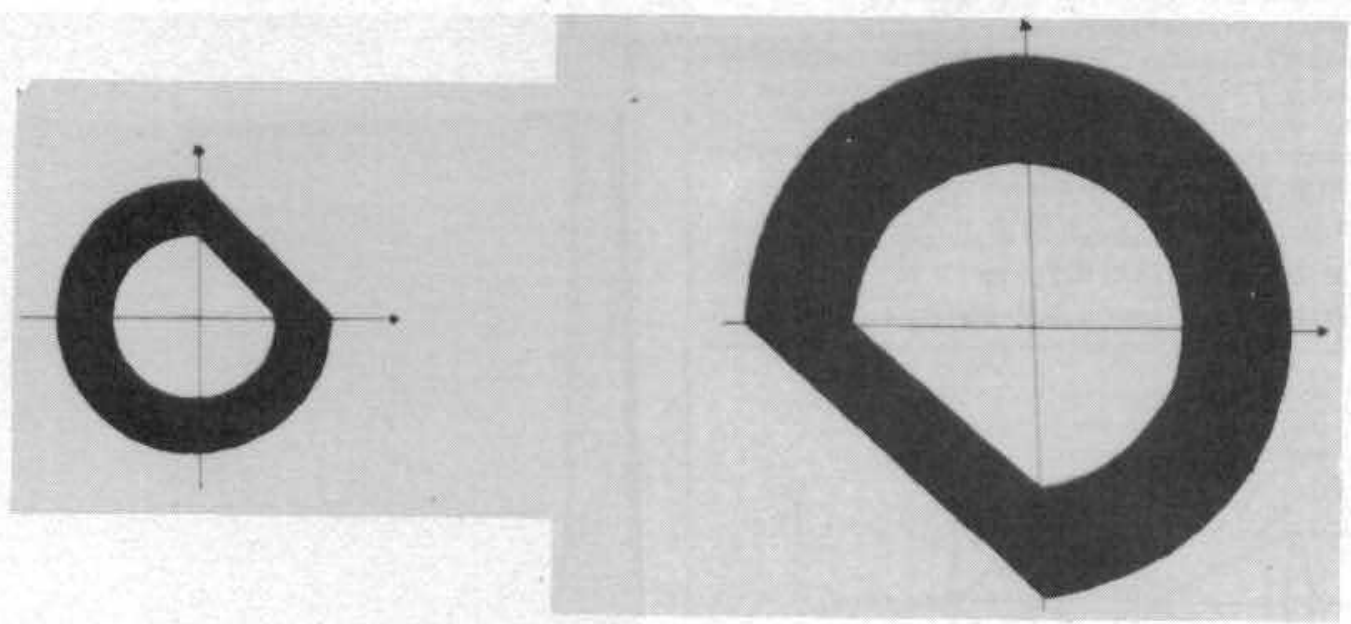

(a)

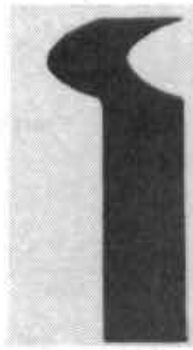

(c)

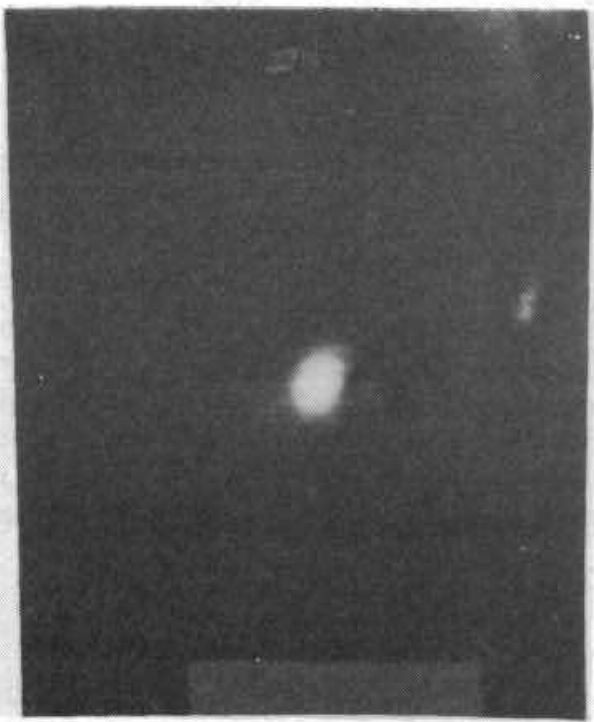

(e) (b)

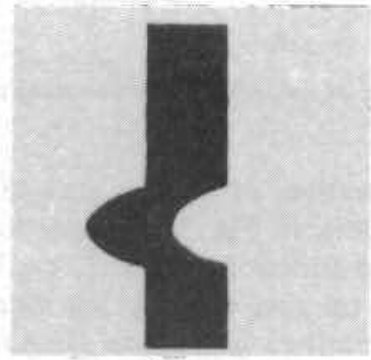

(d)

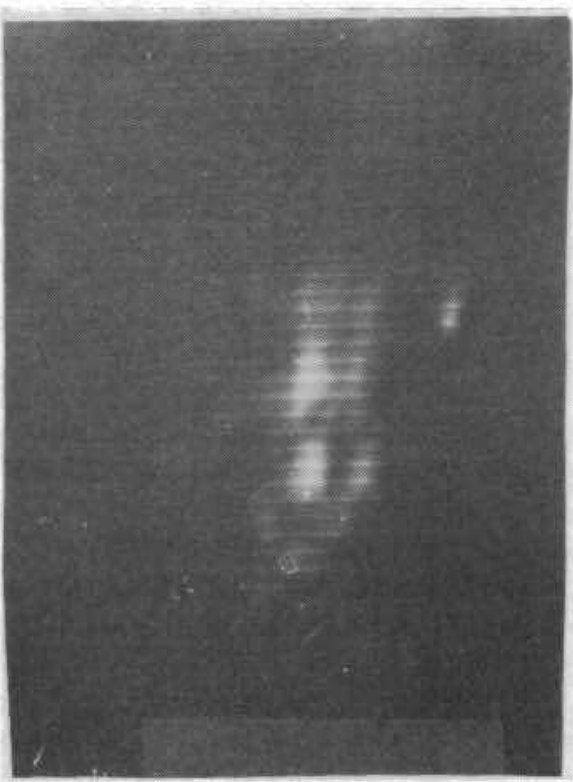

(f)

Fig. 7. Rotational and scale inyariant optical correlation of two input functions that differ in scale by 200 percent and in rotation by $180^{\circ}$. (a) and (b) Inputs. (c) and (d) Polar transformed and logarithmically scaled functions. (e) Autocorrelation of (a). (f) Cross-correlation of both inputs. 